\title{
(1) \\ Intestinal organoids to model cystic fibrosis
}

CrossMark

\author{
Peter van Mourik ${ }^{1}$, Jeffrey M. Beekman ${ }^{1,2}$ and Cornelis K. van der Ent ${ }^{1}$ \\ Affiliations: ${ }^{1}$ Department of Pediatric Pulmonology, Wilhelmina Children's Hospital, University Medical Center \\ Utrecht, Utrecht University, Utrecht, The Netherlands. ${ }^{2}$ Regenerative Medicine Center Utrecht, University \\ Medical Center Utrecht, Utrecht University, Utrecht, The Netherlands.
}

Correspondence: Cornelis K. van der Ent, Dept of Pediatric Pulmonology, Wilhelmina Children's Hospital, University of Utrecht, Lundlaan 6, 3584 EA Utrecht, The Netherlands. E-mail: k.vanderEntवumcutrecht.nl

@ERSpublications

CF can be studied using patient-specific intestinal organoids. CFTR protein function in intestinal organoids correlates with clinical disease severity and drug testing in organoids could aid in finding drugs for people with rare CFTR mutations. https://bit.ly/2GryRl0

Cite this article as: van Mourik P, Beekman JM, van der Ent CK. Intestinal organoids to model cystic fibrosis. Eur Respir J 2019; 54: 1802379 [https://doi.org/10.1183/13993003.02379-2018].

\section{Introduction}

Recent advances in adult stem cell biology have resulted in the development of organoid culture technologies using a variety of tissue sources such as intestine, lung and kidney [1]. Organoids are three-dimensional, multicellular structures that recapitulate tissue features of the parental organ and are usually grown from donor tissue fragments [1]. As organoids are functional expressions of individual genomes, these cultures are particularly useful to understand how genetic factors contribute to individual disease. As such, they are used to study hereditary diseases such as cystic fibrosis (CF), and more common diseases such as cancer where genetics can influence disease severity and drug efficacy $[2,3]$.

Intestinal organoids have been on the forefront of these developments, as culture methodology was first developed for this tissue source [4]. For CF, human intestinal organoids can be grown from intestinal crypt fragments that are isolated from rectal biopsies. Taking rectal biopsies is typically an innocuous procedure that can be performed in all age groups (including newborns) without a need for anaesthesia $[5,6]$. Intestinal organoids can be generated with high individual success rates, which facilitates access to tissue that can be expanded, stored in liquid nitrogen and can be thawed and continuously cultured for $>6$ months while preserving individual functional phenotype [7]. While many labs worldwide have adopted organoid culturing [8], few laboratories have expertise in using intestinal organoids to study CF. Here we focus on applications of intestinal organoids for CF and emphasise important future directions that can help to develop effective therapies for all people with CF.

\section{How does it work?}

Genetic variability of the cystic fibrosis transmembrane conductance regulator (CFTR) gene can result in misfolding and malfunction of the CFTR protein. This subsequently dysregulates epithelial ion and fluid transport, resulting in CF disease manifestations. CFTR plays a dominant role in rapid fluid secretory responses in the large intestine that are evoked by cyclic (c)AMP inducing stimuli, and its activation can lead to secretory diarrhoea. Chloride is the main driver of this rapid fluid secretory response $[9,10]$.

CFTR is expressed on the apical membrane that lines the internal lumen of rectal organoids. CFTR activation by cAMP-raising agents such as forskolin leads to chloride transport into the organoid lumen 
that is accompanied by luminal water secretion through osmosis (figure 1). Two functional assays have been developed to quantify CFTR function in intestinal organoids that rely on the luminal chloride secretion and coupled water transport.

First, incubation of organoids with forskolin leads to rapid luminal fluid secretion through CFTR activation that causes whole organoid swelling within 60 minutes. The forskolin-induced swelling (FIS) phenotype is absent in human and mouse organoids lacking functional CFTR gene products (e.g. two class I mutations or Cftr knockout), and is inhibited by chemical CFTR inhibitors, supporting full CFTR-dependency of the FIS readout [10]. The resulting swelling of organoids is quantified using live confocal microscopy after organoid labelling with calcein green. The relative size increase of all organoids in a well is calculated over time using 10-minute intervals. A relative swelling curve is generated from these data and an area under the curve of this relative swelling is calculated to compare conditions (e.g. different drugs, different donors) in a single graph [11].

Second, steady-state differences in luminal phenotype exist between healthy control and CF organoids independent of forskolin. Healthy control rectal organoids have large fluid-filled lumens, suggesting the presence of functional CFTR and physiological cAMP signalling during standard culture conditions leading to luminal salt and fluid transport [12]. CF organoids do not have lumens that are easily recognised upon visual inspection. This phenotypical difference can be quantified before a FIS assay is performed by manually drawing in the lumen of calcein-labelled organoids in the baseline images, and subsequently expressing the lumen area as percentage of total area. Whereas CF organoids have steady-state lumen areas (SLA) between $0 \%$ and $10 \%$ of total organoid area, healthy control SLA is between $40 \%$ and $80 \%$. While SLA is mostly CFTR-driven, some variability in this phenotype exists at the $0-10 \%$ range independent of CFTR genotype.

FIS and SLA are complementary assays. FIS has considerable throughput and quantifies CFTR function at levels associated with CF disease and treatment thereof with current CFTR modulators [7, 10]. As FIS

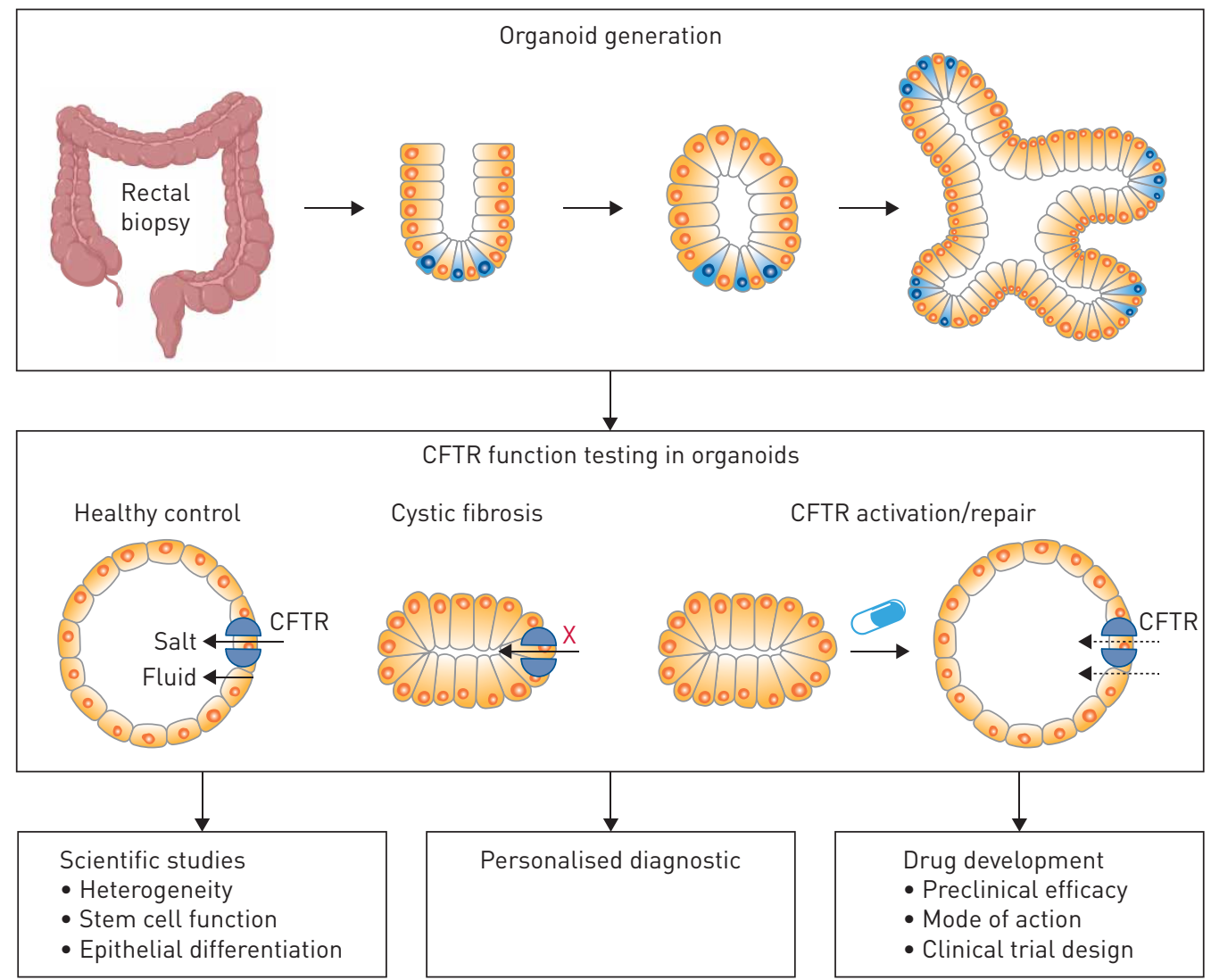

FIGURE 1 Potential applications of intestinal organoids to study cystic fibrosis. Intestinal organoids can be generated from rectal biopsies. In organoids, robust cystic fibrosis transmembrane conductance regulator (CFTR) function measurements can be performed based on phenotypic differences between organoids and the observation that repair and activation of CFTR causes swelling of organoids. This model can be used in preclinical and clinical studies with the potential to obtain patient-specific information on disease severity and CFTR-modulator drug response. Figure partially created using BioRender. 
measures relative size increase, it requires that the initial starting sizes are comparable. This is true for $\mathrm{CF}$ organoids, but at larger SLA $(\sim>25 \%)$, relative size increases are underestimated when two-dimensional area measurements are performed [7]. Therefore, FIS of CF and healthy controls are not directly comparable. SLA facilitates comparison between CF and healthy control organoids, but has limited resolution to discriminate at lower CFTR function levels associated with severe CF disease [7].

Both FIS and SLA have only recently been developed, and technological expertise is currently available in a select number of laboratories. The CFTR dependency of FIS has been confirmed by independent other $\mathrm{CF}[13,14]$ and non-CF laboratories in intestinal [15] and non-intestinal tissues [16]. To further standardise multicentre research, biopsies can also be shipped to a centralised laboratory for processing, which is feasible within $48-72 \mathrm{~h}$ after obtaining the biopsy. Growing organoid structures for FIS experiments takes $\sim 4$ weeks, and at that point organoids can be stored in liquid nitrogen for future use. When in culture, FIS and SLA assays can be performed weekly, and repeated measurements over several weeks can be performed to ensure reliability of measurements.

What is the current use of organoids in studying cystic fibrosis?

Intestinal organoids are used to better understand how CFTR function or manipulation thereof by therapeutic interventions can impact on individual clinical phenotype (figure 1). Additionally, the recently funded HIT-CF project (www.hitcf.org) will use organoids to design clinical trials by pre-treatment stratification of in vitro responders.

Clinical studies indicate that organoid FIS is relevant to characterise CFTR function of rare CFTR variants. Many of the currently known 2000 CFTR variants have not been characterised, which complicates the prediction of individual disease phenotype. Moreover, limited data regarding drug effects on rare genotypes prevents access to existing and new CFTR-mutation specific treatments. Pilot studies indicate that organoids could help both as individual biomarker of CFTR function to predict individual disease phenotype [17], as well as response to therapy [18], which could help characterise rare mutations for treatment response [7].

\section{Organoids and clinical disease phenotype}

Organoid FIS reflects residual CFTR function and correlates with predicted phenotypic characteristics of the CF genotype $[7,17]$ and other biomarkers of CFTR function, such as sweat chloride concentration (SCC) $[7,17]$ and intestinal current measurements (ICM) $[17,19]$. While nasal potential difference (NPD) is another important clinical biomarker of CFTR function, no data on the correlation between organoid FIS and NPD are currently available.

The first study to directly compare organoid FIS and clinical phenotype prospectively included 34 newborns with CF. Newborns were clustered into low FIS or high FIS. Low FIS in organoids was related to increased pulmonary and pancreatic disease parameters at the age of 1 year [17]. Interestingly, in cases where SCC and ICM disagreed, FIS appeared to correctly align with the clinical indicators [17]. This study suggests that the full CFTR dependency of FIS and the 48 datapoints we typically gather (by titrating forskolin and repeating measurements) to type individual CFTR residual function may provide a higher accuracy and precision as compared to other biomarkers of CFTR function. The precise measurement of CFTR function also suggests that a further refinement of residual function classification is possible. Since these early studies are small, it is unclear how well the model predicts long-term clinical risk. Follow-up studies in larger cohorts are needed to validate the use of organoids for predicting disease phenotype in individuals with rare mutations, and predicting phenotypic variability between individuals with identical CF-causing mutations.

\section{Organoids for studying drug efficacy}

Preclinically, organoids are being used to identify and develop CFTR-modulating drugs and explore mechanisms associated with differences in CFTR function. Pharmaceutical companies use intestinal organoids in their drug development pipeline [20,21]. During initial high-throughput screening, many different chemical structures are discovered that could work as CFTR-modulating drugs. Organoids can be a useful tool to efficiently validate lead compounds, because of their medium/high throughput and sensitivity to drug effects in combination with the fact that they express endogenous CFTR. Organoids are used to test the potency of single drugs, but also to compare the efficacy of different combination treatments [20-22].

Moreover, studies by DeKkeRs and co-workers [7, 10, 23] in organoids indicated that both the CFTR mutations and additional patient-specific genetic differences modify response to CFTR modulators. By studying the effects of a range of drugs on different genotypes, the optimal CFTR modulating drug and their potency for each genotype can be identified for further clinical studies [23, 24]. 
Several studies have highlighted the translational potential of organoids. Genotype-specific effects of ivacaftor and lumacaftor/ivacaftor in organoids correlate with clinical trial data at group level [7]. The failure of a phase III clinical trial with ataluren in subjects with stop-codon mutations was preceded by a report on the absence of drug activity in the organoid model $[25,26]$. These group-based observations indicate that organoids can help to characterise the response of mutations to particular treatments. Such an approach would complement current CFTR "theratyping" efforts that mostly rely on cell-line expression systems in which CFTR mutations are introduced. Based on quantitative and qualitative differences in therapy response between organoid subgroups (e.g. comparing average p.Ala455Glu/p. Phe508del, p.Asn1303Lys/p.F508del or class I/p.Phe508del responses), mutation-specific phenotypes with different therapeutic vulnerabilities can be deduced [23]. These theratyping efforts have no direct relationship to the individual patient, but rely on the coupling of individual CFTR genotype to the cell-based data.

Recent proof of concept in two patients with ultra-rare mutations showed clear in vitro-in vivo correlation in response to treatment with ivacaftor [7]. A subsequent study in 24 individuals with a range of genotypes found that intestinal organoid FIS after treatment with the drugs curcumin, genistein, ivacaftor and lumacaftor/ivacaftor correlated with in vivo responses in pulmonary function and sweat chloride concentrations on the individual level. Moreover, receiver operating characteristic curves generated from these data suggest that individual clinical response of CFTR-modulating drugs can be predicted with good-to-high accuracy [18]. These initial studies indicate that organoids provide a functional readout that integrates both the patient-specific CFTR mutations and additional genetic variation that modifies the response to treatment. If these results are validated in subsequent studies, this would suggest that a direct test on the patient organoid can be used to characterise the response to treatment, independent of any $a$ priori knowledge on the CFTR genotype.

\section{How do organoids compare to other ex vivo or in vitro cell models?}

Cell-based approaches have mostly been used to type the average therapeutic response of a mutation or genotype. The Fischer rat thyroid (FRT) cell line, in which CFTR mutations are introduced in a standardised manner using defined cDNAs, can be used for high-throughput drug screening, and has helped to extend ivacaftor treatment to new CFTR mutations [27]. The strength of this model is its definition of standardised test components, and that patients do not have to undergo additional procedures. However, FRT cells are nonhuman, which influences CFTR protein folding [28] and pharmacological treatment responsiveness $[29,30]$. To complement this mutation-oriented approach, several models have been developed using patient-derived cells.

Only a few studies directly compare drug outcomes in ex vivo or in vitro cultured cells and in vivo parameters of treatment. ICM on ex vivo rectal biopsies is validated for measuring residual CFTR function $[31,32]$, and sensitively detects CFTR-modulator treatment effects after in vivo drug treatment [33, 34]. However, only one therapy can be tested per biopsy, making personalised drug assessment burdensome.

CFTR function in human bronchial epithelial (HBE) and nasal epithelial (HNE) cells is assessed using electrophysiological studies on air-liquid interface cultures, which mimics the airway environment [35]. High-throughput screening is not feasible due to cell senescence after a limited number of passages, and repeatedly obtaining HBE cells from living donors is an invasive process. HNE cells can be obtained by nasal brushing, and initial studies show that electrophysiological measurements in brushed HNE cells correlate with in vivo CFTR function and might be predictive of individual treatment response [36, 37].

Compared to the aforementioned models, intestinal organoids have both limitations and advantages. Because healthy organoids are pre-swollen, FIS cannot compare CF to healthy control CFTR function. Moreover, although both intestinal and airway cells are derived from the endoderm, it is unclear whether intestinal epithelial CFTR function is directly related to airway epithelial CFTR function, which might influence treatment response $[29,30]$. Nevertheless, the potential to biobank and repeatedly test intestinal organoids facilitates robust personalised medicine approaches. Future studies are needed to elucidate which model is superior in terms of feasibility and predictive capacity.

NPD and SCC are established in vivo CFTR function measurements that are both used for diagnosis of CF and as a biomarker in phase I and II clinical trials to evaluate CFTR function improvement after drug treatment [38]. These methods have been thoroughly validated and are available in many centres. However, NPD and SCC cannot be used preclinically, which limits their use for personalised medicine approaches. For an in-depth overview of studies on ICM, NPD and SCC see DE BoECK et al. [39].

\section{Future use of organoids}

Current studies with organoids support the possibility of additional classification of residual function. CFTR function is probably a biological continuum that is mostly dependent on CFTR genotype and 
further modified by individual genetic and environmental factors. The first clinical validation studies showed how relatively large differences in residual function (albeit all in the CF domain) correspond with clinical manifestations [17]. Whether more subtle differences in organoid swelling (e.g. between organoids from people with identical CF-causing mutations; or in conditions of borderline CF and CFTR-related disease) also associate with clinical phenotype still need further long-term clinical follow-up studies.

The current focus of organoid studies in CF is on pretreatment selection of patients with ultra-rare CFTR variants in a Europe-wide consortium study (www.hitcf.org). Additionally, this study highlights the possibility of using the organoid technology to study pharmacy-overarching drug combinations. Future studies should define more precisely how assay sensitivity and specificity change in relation to the magnitude of treatment effects in vivo and how short term predictions relate to long-term outcomes. Implementing organoids in dose-optimisation studies might help to further individualise treatment with upcoming CFTR-modulating drugs.

\section{Conclusion}

Rectal organoids are in vitro primary cell cultures that enable the individual typing of CFTR function and response to CFTR modulators. The first clinical studies indicate that FIS of rectal organoids help to characterise the disease phenotype and drug response of rare CFTR mutations. Additional studies are underway to further validate relations between in vitro CFTR function and in vivo clinical phenotype in the context of CF diagnosis, prognosis and therapeutic response.

Conflict of interest: P. van Mourik has nothing to disclose. J.M. Beekman has a patent on organoid swelling for personalised diagnosis in CF with royalties paid by Hubrecht Organoid Technology. He received financial support to cover travel costs and preparation time of scientific lectures from Vertex. He also received funding from Galapagos for CFTR modulator studies. C.K. van der Ent reports grants from Vertex Pharmaceuticals Inc, Gilead Sciences and Galapagos, grants and other funding from ProQR, and other funding from TEVA pharmaceutical industries, outside the submitted work.

\section{References}

$1 \quad$ Clevers H. Modeling development and disease with organoids. Cell 2016; 165: 1586-1597.

2 Sachs N, de Ligt J, Kopper O, et al. A living biobank of breast cancer organoids captures disease heterogeneity. Cell 2018; 172: 373-386.

3 Drost J, Clevers H. Organoids in cancer research. Nat Rev Cancer 2018; 18: 407-418.

4 Sato T, Stange DE, Ferrante M, et al. Long-term expansion of epithelial organoids from human colon, adenoma, adenocarcinoma, and Barrett's epithelium. Gastroenterology 2011; 141: 1762-1772.

5 Friedmacher F, Puri P. Rectal suction biopsy for the diagnosis of Hirschsprung's disease: a systematic review of diagnostic accuracy and complications. Pediatr Surg Int 2015; 31: 821-830.

6 Servidoni MF, Sousa M, Vinagre AM, et al. Rectal forceps biopsy procedure in cystic fibrosis: technical aspects and patients perspective for clinical trials feasibility. BMC Gastroenterol 2013; 13: 91.

7 Dekkers JF, Berkers G, Kruisselbrink E, et al. Characterizing responses to CFTR-modulating drugs using rectal organoids derived from subjects with cystic fibrosis. Sci Transl Med 2016; 8: 344ra84.

8 Wallach TE, Bayrer JR. Intestinal organoids: new frontiers in the study of intestinal disease and physiology. J Pediatr Gastroenterol Nutr 2017; 64: 180-185.

9 Thiagarajah JR, Broadbent T, Hsieh E, et al. Prevention of toxin-induced intestinal ion and fluid secretion by a small-molecule CFTR inhibitor. Gastroenterology 2004; 126: 511-519.

10 Dekkers JF, Wiegerinck CL, de Jonge HR, et al. A functional CFTR assay using primary cystic fibrosis intestinal organoids. Nat Med 2013; 19: 939-945.

11 Boj SF, Vonk AM, Statia M, et al. Forskolin-induced swelling in intestinal organoids: an in vitro assay for assessing drug response in cystic fibrosis patients. J Vis Exp 2017; 120: e55159.

12 Riordan JR, Rommens JM, Kerem B, et al. Identification of the cystic fibrosis gene: cloning and characterization of complementary DNA. Science 1989; 245: 1066-1073.

13 Awatade NT, Ramalho S, Silva IAL, et al. R560S: a class II CFTR mutation that is not rescued by current modulators. J Cyst Fibros 2019; 18: 182-189.

14 Clarke LA, Awatade NT, Felício VM, et al. The effect of premature termination codon mutations on CFTR mRNA abundance in human nasal epithelium and intestinal organoids: a basis for read-through therapies in cystic fibrosis. Hum Mutat 2019; 40: 326-334.

15 Foulke-Abel J, In J, Yin J, et al. Human enteroids as a model of upper small intestinal ion transport physiology and pathophysiology. Gastroenterology 2016; 150: 638-649.

16 Sachs N, Papaspyropoulos A, Zomer-van Ommen DD, et al. Long-term expanding human airway organoids for disease modeling. EMBO J 2019; 38: e100300.

17 de Winter-de Groot KM, Janssens HM, van Uum RT, et al. Stratifying infants with cystic fibrosis for disease severity using intestinal organoid swelling as a biomarker of CFTR function. Eur Respir J 2018; 52: 1702529.

18 Berkers G, van Mourik P, Vonk AM, et al. Rectal organoids enable personalized treatment of cystic fibrosis. Cell Rep 2019; 26: 1701-1708.

19 Zomer-van Ommen DD, de Poel E, Kruisselbrink E, et al. Comparison of ex vivo and in vitro intestinal cystic fibrosis models to measure CFTR-dependent ion channel activity. J Cyst Fibros 2018; 17: 316-324.

20 Musch S, Ramalho A, Van Der Plas S, et al. Poster Session Abstracts. Pediatr Pulmonol 2016; 51: S194-S485.

21 Kolodziej A. Fixing $\Delta$ F508-CFTR: Bringing New Correctors into the Fold. www.flatleydiscoverylab.com/ wp-content/uploads/2017/07/Fixing- $\Delta$ F508-CFTR---Bringing-New-Correctors-into-the-Fold.pdf Date last accessed: January 23, 2019. Date last updated: 2017. 
22 Dekkers JF, Van Mourik P, Vonk AM, et al. Potentiator synergy in rectal organoids carrying S1251N, G551D, or F508del CFTR mutations. J Cyst Fibros 2016; 15: 568-578.

23 Dekkers JF, Gogorza Gondra Ra, Kruisselbrink E, et al. Optimal correction of distinct CFTR folding mutants in rectal cystic fibrosis organoids. Eur Respir J 2016; 48: 451-458.

24 Vijftigschild LAW, Berkers G, Dekkers JF, et al. $\beta_{2}$-Adrenergic receptor agonists activate CFTR in intestinal organoids and subjects with cystic fibrosis. Eur Respir J 2016; 48: 768-779.

25 Zomer-van Ommen DD, Vijftigschild LAW, Kruisselbrink E, et al. Limited premature termination codon suppression by read-through agents in cystic fibrosis intestinal organoids. J Cyst Fibros 2016; 15: 158-162.

26 PTC Therapeutics. PTC Therapeutics Announces Results from Pivotal Phase 3 Clinical Trial of Ataluren in Patients Living with Nonsense Mutation Cystic Fibrosis. http://ir.ptcbio.com/news-releases/news-release-details/ ptc-therapeutics-announces-results-pivotal-phase-3-clinical?ReleaseID $=1015471$ Date last accessed: March 28, 2018. Date last updated: March 2, 2017.

27 US Food and Drug Administration (FDA). FDA Expands Approved use of Kalydeco to Treat Additional Mutations of Cystic Fibrosis. 2017. www.fda.gov/NewsEvents/Newsroom/PressAnnouncements/ucm559212.htm Date last accessed: March 28, 2018. Date last updated: May 17, 2017.

28 Wang X, Koulov A V, Kellner WA, et al. Chemical and biological folding contribute to temperature-sensitive $\Delta$ F508 CFTR trafficking. Traffic 2008; 9: 1878-1893.

29 Pedemonte N, Tomati V, Sondo E, et al. Influence of cell background on pharmacological rescue of mutant CFTR. Am J Physiol Cell Physiol 2010; 298: C866-C874.

30 Rowe SM, Pyle LC, Jurkevante A, et al. $\triangle$ F508 CFTR processing correction and activity in polarized airway and non-airway cell monolayers. Pulm Pharmacol Ther 2010; 23: 268-278.

31 Hirtz S, Gonska T, Seydewitz $\mathrm{HH}$, et al. CFTR $\mathrm{Cl}^{-}$channel function in native human colon correlates with the genotype and phenotype in cystic fibrosis. Gastroenterology 2004; 127: 1085-1095.

32 Sousa M, Servidoni MF, Vinagre AM, et al. Measurements of CFTR-mediated $\mathrm{Cl}^{-}$secretion in human rectal biopsies constitute a robust biomarker for cystic fibrosis diagnosis and prognosis. PLoS One 2012; 7: e47708.

33 Graeber SY, Hug MJ, Sommerburg O, et al. Intestinal current measurements detect activation of mutant CFTR in patients with cystic fibrosis with the G551D mutation treated with ivacaftor. Am J Respir Crit Care Med 2015; 192: $1252-1255$.

34 Graeber SY, Dopfer C, Naehrlich L, et al. Effects of lumacaftor-ivacaftor therapy on cystic fibrosis transmembrane conductance regulator function in Phe508del homozygous patients with cystic fibrosis. Am J Respir Crit Care Med 2018; 197: 1433-1442.

35 Neuberger T, Burton B, Clark $\mathrm{H}$, et al. Use of primary cultures of human bronchial epithelial cells isolated from cystic fibrosis patients for the pre-clinical testing of CFTR modulators. Methods Mol Biol 2011; 741: 39-54.

36 Brewington JJ, Filbrandt ET, LaRosa FJ, et al. Brushed nasal epithelial cells are a surrogate for bronchial epithelial CFTR studies. JCI Insight 2018; 3; 99385.

37 Pranke IM, Hatton A, Simonin J, et al. Correction of CFTR function in nasal epithelial cells from cystic fibrosis patients predicts improvement of respiratory function by CFTR modulators. Sci Rep 2017; 7: 7375.

38 Ramsey BW, Davies J, McElvaney G, et al. A CFTR potentiator in patients with cystic fibrosis and the G551D mutation. N Engl J Med 2011; 365: 1663-1672.

39 De Boeck K, Kent L, Davies J, et al. CFTR biomarkers: time for promotion to surrogate end-point? Eur Respir J 2013; 41: 203-216. 